\title{
Prompt photon production in deep inelastic scattering at HERA
}

\section{Olena Hlushchenko*}

National Taras Shevchenko University of Kyiv

E-mail: olena.hlushchenko@desy.de

for the ZEUS Collaboration

Prompt photon production combined variables in deep inelastic scattering have been studied using the ZEUS detector during the HERAII running period. The total integrated luminosity of the used data sample is $326 \mathrm{pb}^{-1}$. The differential cross sections are compared to the MC and theoretical predictions at the parton level.

XXIV International Workshop on Deep-Inelastic Scattering and Related Subjects

11-15 April, 2016

DESY Hamburg, Germany

${ }^{*}$ Speaker. 


\section{Introduction}

The subject of this talk is a continuation of a previous study of prompt photon production in $e p$ deep inelastic scattering (DIS). Outgoing photons with high transverse energy, $E_{T}>4 \mathrm{GeV}$ , may be radiated either by a scattered lepton (LL photons) or by a parton (QQ photons), see Fig.1. A requirement that the observed photon is isolated in the event reduces the backgrounds from decay products of a high energy hadron and photons from an outgoing high- $E_{T}$ quark which is problematic to calculate [2].

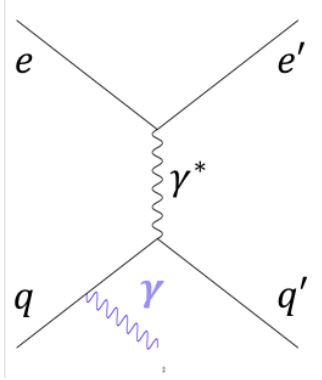

(a)

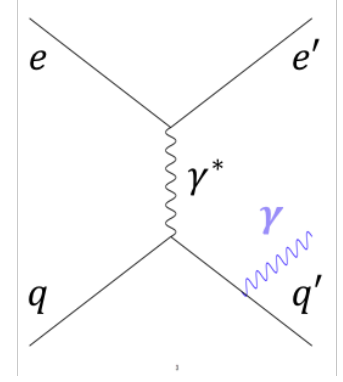

(b)

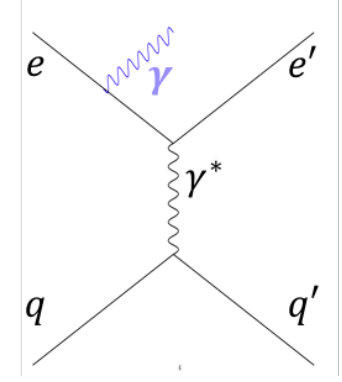

(c)

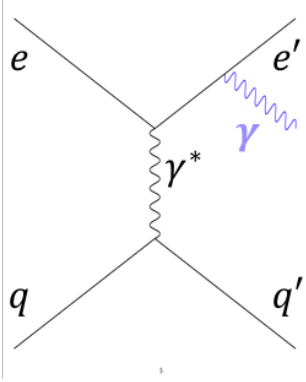

(d)

Figure 1: Lowest-order diagrams for photon production in ep scattering. (a) - (b): quark radiative diagrams; (c) - (d): lepton radiative diagrams.

Prompt photons are produced directly from the partonic interaction and in this way they provide a good tool for both QCD and QED tests and can give an additional check of the proton's parton distribution functions. Compared to the studies of final hadronic states, photons do not change their properties before the observation in the detector since they are not affected by the hadronisation, unlike the partons that form jets. Study of the dynamics of prompt photon emission can be used to probe different theoretical models. One also can observe how the dynamics changes with virtuality scale by comparing new results with the corresponding results for photoproduction [1]. Additionally photons are considered as a possible background to new physics processes.

\section{Apparatus and Event selection}

For the measurement, a combined data sample, recorded using the ZEUS detector at HERA, with both $e^{+} p$ and $e^{-} p$ events and integrated luminosity of $326 \mathrm{pb}^{-1}$ was used. The lepton and proton beam energy were respectively $E_{e}=27.5 \mathrm{GeV}$ and $E_{p}=920 \mathrm{GeV}$. The detailed description of ZEUS detector can be found elsewhere [3].

The principal component of the ZEUS detector used in this analysis was a high-resolution uranium-scintillator calorimeter (CAL). The calorimeter was divided into three regions, forward (FCAL), barrel (BCAL) and rear(RCAL), and each region consisted of a finely segmented electromagnetic section outside which was a hadronic section with larger cells. The "forward" (Z) direction is the proton beam direction. The fine granularity along the beam axis $(5 \mathrm{~cm}$ per cell) of the electromagnetic calorimeter of the BCAL (BEMC) allowed a good separation of signal photons from the neutral hadrons decay background. Photons from reactions such as $\pi_{0} \rightarrow \gamma \gamma$ have 
in general wider spread along the axis and often hit few neighbouring cells while signal photon hits a single cell. The cut on the width of the signal was chosen in the way background could be evaluated.

The basic event selection and reconstruction were performed as previously [4]. The most important physics selections are as follows:

\section{- Prompt photon selection}

$4<E_{T}^{\gamma}<15 \mathrm{GeV}$ - transverse energy of the prompt photons candidate

$0.7<\eta^{\gamma}<0.9 \quad$ - pseudorapidity of the prompt photon candidate (detected in BCAL)

$\frac{E_{E M C}}{E_{E M C}+E_{H A D}}>0.9$ - at least $90 \%$ of the candidate's energy is deposited in BEMC

$\Delta R(\eta, \phi)<0.2 \quad$ - no other tracks within a narrow cone in the $\eta-\phi$ plane (isolation cut). Here $\Delta R(\eta, \phi)=\sqrt{(\Delta \phi)+(\Delta \eta)^{2}}$

$\frac{E^{\gamma}}{E^{j e t ~ w i t h ~} \gamma}>0.9 \quad$ - at least $90 \%$ of the reconstructed jet energy is carried by prompt photon candidate

\section{- Accompanying jet selection}

$E_{T}^{\text {jet }}>2.5 \mathrm{GeV} \quad$ - transverse energy of the jet

$1.5<\eta^{\gamma}<1.8 \quad$ - pseudorapidity of the jet

$E_{m a x}^{j e t} \quad-$ the jet that has a highest reconstructed energy is considered as accompanying

\section{- Kinematics selection}

$$
\begin{array}{ll}
10<Q^{2}<350 \mathrm{GeV}^{2} & \text { - energy of the prompt photons candidate } \\
E^{e}>10 \mathrm{GeV} & \text { - energy of the scattered lepton } \\
140^{\circ}<\theta^{e}<180^{\circ} & \text { - electron scattering angle } \\
35<E-p_{Z}<65 \mathrm{GeV} & \text { - this is calculated from the recorded energy in the detector, and } \\
& \begin{array}{l}
\text { corresponds to roughly twice the energy of the incoming electron, } \\
\text { showing that the event is well recorded. }
\end{array}
\end{array}
$$

Jets were reconstructed using the $k_{T}$ clustering algorithm with the radius parameter set to 1.0, making use of energy-flow objects (EFO's), constructed from clusters of calorimeter cells and tracks, and excluding the scattered electron.

To extract the photon signal from the background, the quantity $\langle\delta Z\rangle$, defined as the $E_{T}$ weighted mean of the distance of the $Z$ position of the electromagnetic cells in the cluster relative to the mean $Z$ of the cluster: $\langle\delta Z\rangle=\sum_{i} E_{i}\left|Z_{i}-Z_{\text {cluster }}\right| /\left(w_{\text {cell }} \sum_{i} E_{i}\right)$, was used as described in previous ZEUS analyses $[4,5,6,7,8,9]$. Here, $Z_{i}$ is the $Z$ position of the centre of the $i$-th cell, $Z_{\text {cluster }}$ is the centroid of the EFO cluster, $w_{\text {cell }}$ is the width of the cell in the $Z$ direction, and $E_{i}$ is the energy recorded in the cell. The sum runs over all BEMC cells in the EFO. 
The total $\langle\delta Z\rangle$ distribution is shown in Fig.2 plotted in units of electromagnetic calorimeter cell widths. The MC samples corresponding to different sources of high$E_{T}$ photons were scaled to the level of data luminosity. The LL component (generated using ARIADNE 4.12 [10]) of the signal was extracted from the data before the fit, in which only one parameter was varied the fraction of QQ (generated using PYTHIA 6.416 [11]) component in the data after the extraction of LL component from it. A peak at low $\langle\delta Z\rangle$ indicates that most of the energy is found in one cell - actual prompt photons. The broader background peaks around a value of approximately 0.5 , where the cluster energy is shared between two contiguous cells - hadronic background. Fits are performed to the data in each bin of each quantity in the range $0.05<\langle\delta Z\rangle<0.8$ whose cross section is to be measured, to extract the photon signal.

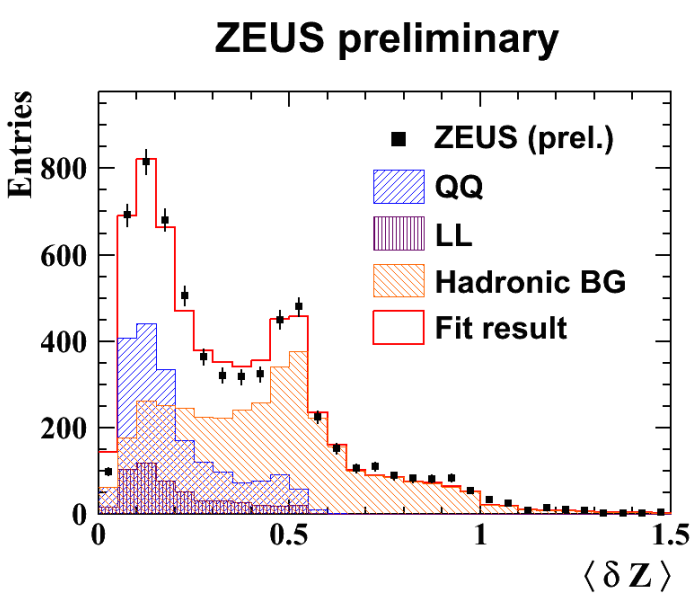

Figure 2: Distribution of $\langle\delta Z\rangle$ for the entire selected data sample. The error bars represent the statistical uncertainties. The red line shows a fit to the data of three components with fixed shapes as described in the text. The blue shaded histogram represents the QQ component of the fit, and the purple histogram - the LL component.

For this paper a set of six combined variables was studied. They are defined as follows:

- the variable $x_{\gamma}$ is a measure of the fraction of the incoming photon energy and momentum that is given to the photon and the jet:

$$
x_{\gamma}=\frac{E^{\gamma}-p_{z}^{\gamma}+E^{j e t}-p_{z}^{j e t}}{2 E_{e} y_{J B}},
$$

where $E^{\gamma}$ and $E^{j e t}$ denote the energies of the photon and the jet, respectively, $p_{z}$ denotes the corresponding longitudinal momenta, $E_{e}=27.5 \mathrm{GeV}, y_{J B}=\sum\left(E-p_{z}\right) / 2 E_{e}$, summing over all energy-flow objects in the event, each treated as equivalent to a massless particle;

- the kinematic quantity $x_{p}$ equals to the fraction of proton energy taken by the parton that interacts with the photon:

$$
x_{p}=\frac{E^{\gamma}+p_{z}^{\gamma}+E^{j e t}+p_{z}^{j e t}}{2 E_{p}},
$$

where $E_{p}=920 \mathrm{GeV}$. This distribution is sensitive to the partonic structure of the proton;

- $\Delta \phi$ is the azimuthal angle between the prompt photon and the jet: $\Delta \phi=\phi^{j e t}-\phi^{\gamma}$, where $\phi^{\gamma}$ and $\phi^{j e t}$ denote azimuthal angles of the photon and the jet, respectively. This variable is sensitive to the presence of higher-order gluon radiation relative to the outgoing quark, which can generate non-collinearity between the photon and the leading jet; 
- $\Delta \eta$ is the pseudorapidity difference between the prompt photon and the jet: $\Delta \eta=\eta^{\text {jet }}$ $\eta^{\gamma}$, where $\eta^{\gamma}$ and $\eta^{\text {jet }}$ denote pseudorapidity of the photon and the jet, respectively. The pseudorapidity is sensitive to the spin of the exchanged quantum [13];

- $\Delta \phi^{e, \gamma}$ is the azimuthal angle between the prompt photon and the scattered electron: $\Delta \phi^{e, \gamma}=$ $\phi^{e}-\phi^{\gamma}$, where $\phi^{\gamma}$ and $\phi^{e}$ denote azimutal angles of the photon and the electron, respectively;

- $\Delta \eta^{e, \gamma}$ is the pseudorapidity difference between the prompt photon and the scattered electron: $\Delta \eta^{e, \gamma}=\eta^{e}-\eta^{\gamma}$, where $\eta^{\gamma}$ and $\eta^{e}$ denote pseudorapidity of the photon and the scattered electron, respectively;

A similar analysis was previously done for photoproduction [12] for all variables except the last two, since the scattered electron is unobservable in photoproduction.

\section{Results}

The cross sections were calculated using acceptance corrections calculated on a bin-by-bin basis. Systematic uncertainties are dominated by the photon energy scale and the modelling of the background.

Preliminary resulting DIS cross sections as functions of $0<x_{\gamma}<1,0<x_{p}<0.07,0^{\circ}<\Delta \phi<$ $180^{\circ},-2.2<\Delta \eta<2,0^{\circ}<\Delta \phi_{e, \gamma}<180^{\circ}$ and $-3.6<\Delta \eta_{e, \gamma}<-0.6$ are shown in Fig.3 and Fig.4. For these figures, the MC QQ cross section was scaled by a factor of 1.6, as in previous publication. After the scaling MC shows a good agreement in shape with the data.

Comparison is made with the model of Baranov et al., [2], in which unintegrated parton densities are used in a LO QCD framework, see Fig.4. The theory predictions are at the parton level. There is reasonable agreement between the experimental data and the shapes of the model distributions (except for $x_{\gamma}$ ), but the absolute cross sections are not well predicted by the BLZ model.

\section{References}

[1] H. Abramowicz et al. [ZEUS Collaboration], Phys. Lett. B 730 (2014) 293, DESY-13-234, arXiv:1312.1539 [hep-ex].

[2] S.P. Baranov, A.V. Lipatov, N.P. Zotov, Phys. Rev. D 81 (2010) 094034 [arXiv:1001.4782 [hep-ph]].

[3] ZEUS Collaboration, U. Holm (ed.), The ZEUS Detector. Status Report (unpublished), DESY (1993), available on http://www-zeus.desy.de/bluebook/bluebook.html

[4] H. Abramowicz et al. [ZEUS Collaboration], Phys. Lett. B 715 (2012) 88, DESY-12-089, arXiv:1206.2270 [hep-ex]

[5] J. Breitweg et al. [ZEUS Collaboration], Phys. Lett. B 413 (1997) 201

[6] J. Breitweg et al. [ZEUS Collaboration], Phys. Lett. B 472 (2000) 175

[7] S. Chekanov et al. [ZEUS Collaboration], Phys. Lett. B 511 (2001) 19

[8] S. Chekanov et al. [ZEUS Collaboration], Phys. Lett. B 595 (2004) 86

[9] S. Chekanov et al. [ZEUS Collaboration], Phys. Lett. B 687 (2010) 16 
[10] L. Lönnblad, Ariadne version 4 - A program for simulation of QCD cascades implementing the colour dipole model, Comp. Phys. Comm, 71 (1992) 15.

[11] T. Sjöstrand et al., PYTHIA 6.4 physics and manual, JHEP 0605 (2006) 26.

[12] H. Abramowicz et al. [ZEUS Collaboration], JHEP 08 (2014) 23

[13] M. Derrick et al. [ZEUS Collaboration], Phys. Lett. B 384 (1996) 401 


\section{ZEUS preliminary}
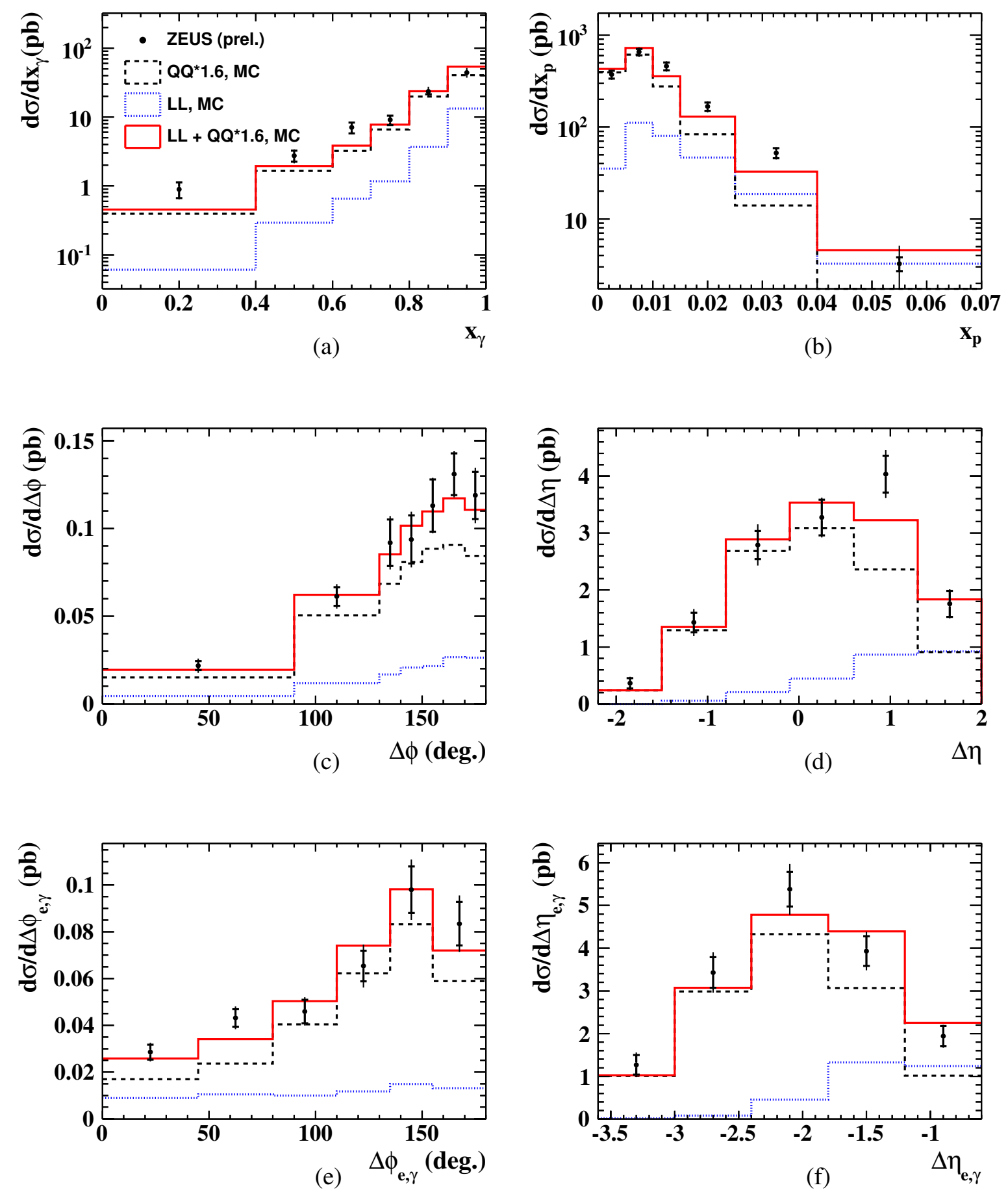

Figure 3: Differential cross sections in (a) $x_{\gamma}$, (b) $x_{p}$, (c) $\Delta \phi$ (deg.), (d) $\Delta \eta$, (e) $\Delta \phi_{e, \gamma}$ (deg.), and (f) $\Delta \eta_{e, \gamma}$. The inner and outer error bars show, respectively, the statistical uncertainty and the statistical and systematic uncertainties added in quadrature. The solid histograms are the reweighted Monte Carlo predictions from the sum of QQ photons from PYTHIA normalized by a factor 1.6 plus DJANGOH LL photons. The dashed (dotted) lines show the QQ (LL) contributions. 


\section{ZEUS preliminary}
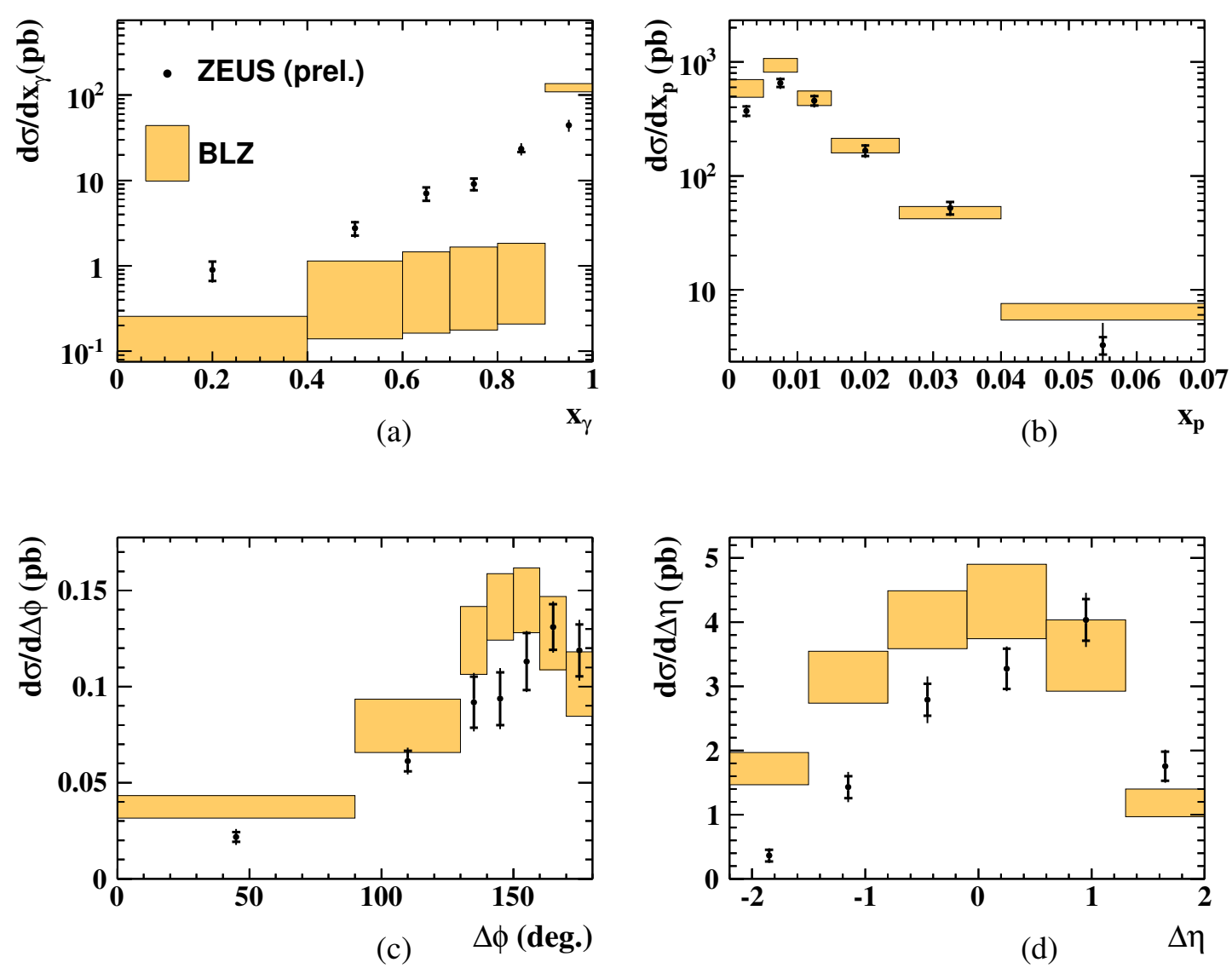

(d)
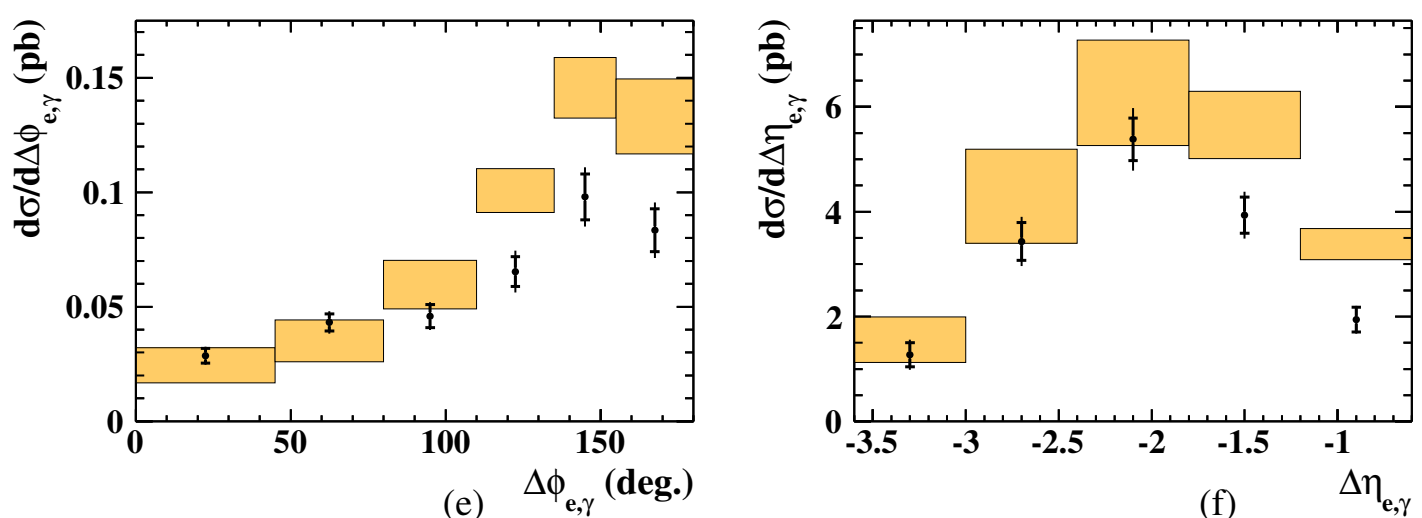

Figure 4: Data points as shown in Fig. 3. Theoretical predictions from Baranov et al. (BLZ) [2] are shown, with associated uncertainties indicated by the shaded bands. 